\title{
Kernos
}

Revue internationale et pluridisciplinaire de religion grecque antique

$17 \mid 2004$

Varia

\section{Nicole BELAYCHE, Simon C. MIMOUNI (dir.), Les communautés religieuses dans le monde gréco- romain. Essais de définition}

\section{Catherine Lheureux-Godbille}

\section{OpenEdition}

Journals

Édition électronique

URL : http://journals.openedition.org/kernos/1510

DOI : 10.4000/kernos. 1510

ISSN : 2034-7871

\section{Éditeur}

Centre international d'étude de la religion grecque antique

\section{Édition imprimée}

Date de publication : 1 janvier 2004

ISSN : 0776-3824

\section{Référence électronique}

Catherine Lheureux-Godbille, « Nicole BeLAyche, Simon C. mimouni (dir.), Les communautés religieuses dans le monde gréco-romain. Essais de définition », Kernos [En ligne], 17 | 2004, mis en ligne le 16 juin 2011, consulté le 21 septembre 2020. URL : http://journals.openedition.org/kernos/1510 ; DOI : https://doi.org/10.4000/kernos.1510 
Nicole Belayche, Simon C. Mimouni (dir.), Les communautés religieuses dans le monde gréco-romain. Essais de définition, Turnhout, Brepols Publishers, 2003. 1 vol. $15,5 \times 24 \mathrm{~cm}, 351$ p. (Bibliothèque de l'École des Hautes Études. Sciences religieuses, 117. Centre d'étude des religions du livre). ISBN : 2-50352204-1.

Ainsi que le remarque Nicole Belayche dans l'introduction de cet ouvrage, la volonté de rendre compte des sociétés gréco-romaines dans leur dimension communautaire se heurte d'emblée au double problème de la définition que l'on donne au terme communauté et de la méthode que l'on va utiliser pour approcher cette réalité. La grande diversité des communautés de toutes natures et des termes qui servent à les désigner accrô̂t encore la difficulté de l'élaboration d'outils de travail : la classification selon le critère de l'intervention de références religieuses dans l'activité du groupe s'avère en effet le plus souvent insuffisante. Il est également très difficile de cadrer les communautés avec précision à travers l'examen des différents marqueurs rituels, conceptuels, comportementaux, hiérarchiques et sociaux, tant ils sont peu spécifiques de l'une ou l'autre forme d'association. L'approche terminologique proposée par Simon Mimouni complète ces quelques mots introductifs.

La première partie de l'ouvrage est consacrée à la recherche d'éléments de définition dans différents contextes associatifs. Dans la première contribution, Anne Logeay s'intéresse, à travers le regard posé par Sénèque sur les comportements religieux de son temps, aux motivations qui sous-tendent la formation des communautés et à l'attitude exemplaire que doit adopter le sage stoïcien face aux débordements découlant inévitablement de l'existence de ces groupes. Dans le deuxième exposé, William Van Andringa s'écarte de la perspective religieuse pour entrer plus directement sur le terrain de la vie clans les cités, à travers l'examen des conventus regroupant les citoyens romains à l'extérieur de leur cité d'origine. La troisième communication est consacrée par John Scheid à la reprise du problème de la définition de la communauté dans l'Antiquité depuis les premiers jalons posés par Theodor Mommsen au milieu du $\mathrm{XTX}^{c}$ siècle : l'observation de pratiques rituelles dans toutes les communautés, la difficulté d'établir alors une distinction claire entre les communautés à vocation religieuse et celles visant plus essentiellement un objectif de vie collective et le caractère fragmentaire des sources quant à la réalité des motivations animant les groupes amènent à séparer la structure communautaire du fait religieux dans la recherche d'une définition. Les deux exposés suivants, de Marie-Françoise Baslez et de Tessa Rajak, ainsi que celui de Nicole Belayche clôturant cette première partie, proposent une approche nuancée des communautés juives évoluant dans un environnement grécoromain où la religion païenne est prédominante. A travers la définition du type de communauté connue par les manuscrits de Qumrân sous le nom de yabad, MarieFrançoise Baslez présente un schéma organisationnel novateur à la charnière des conceptions juives et grecques; Tessa Rajak se livre à l'examen des modalités de contact entre les communautés juives et les autres membres du corps social dans le cadre de la vie civique en Asie Mineure : le biais choisi est l'étude des modes de cohabitation au sein des cités à population mixte et d'acculturation des communautés juives, permettant de concilier la volonté juive de préservation des traditions religieuses et la nécessité pour l'autorité romaine d'assurer l'ordre civil. Enfin, suivant les directions de recherche proposées dans l'introduction par Simon Mimouni, Alain Le Boulluec se penche sur les problèmes terminologiques posés par l'élaboration des premiers discours hérésiologiques en contexte chrétien, mettant en évidence le caractère dynamique de ces représentations destinées à exclure. 
Dans la seconde partie de l'ouvrage, les différents contributeurs s'intéressent plus particulièrement aux marqueurs des communautés religieuses. Les éléments pris en compte dans les différents chapitres constituent de véritables pierres de touche pour la construction d'une définition. Le sujet traité par Emmanuelle Main, la question de la résurrection des morts dans les polémiques entre pharisiens et sadducéens, permet d'approcher concrètement le statut de la Torah à l'intérieur des groupes composant la communauté juive. La comparaison, établie par Simon Nimouni, du rite d'eau en vigueur dans les communautés pharisiennes et du baptême pratiqué par les groupes chrétiens, affine l'analyse traditionnelle qui les fait découler l'un de l'autre par la mise en évidence d'une filiation commune à un rituel judaïque plus ancien. Spécialiste des relations entre les communautés juives et les premiers groupes jucléo-chrétiens, Liliane Vana présente une approche nuancée de la Birkat ha-minim qui constitue non pas une prière dirigée particulièrement contre les judéo-chrétiens, mais plutôt un serment fédérateur destiné à assurer la cohésion des communautés juives contre tout ce qui est susceptible de menacer le processus d'harmonisation et d'uniformisation amorcé au I ${ }^{\text {ur }}$ siècle ap. J.-C. par Rabban Gamliel II. René Sigmund Bloch propose ensuite une approche plus précise des textes consacrés à la magie juive par Flavius Josèphe qui tente de présenter cet aspect problématique de la pratique juive à travers le filtre d'une interpretatio Romana. L'étude d'Éric Rebillard sur l'élection des lieux de sépulture en relation avec les différentes affiliations religieuses aboutit à la conclusion étonnante de l'absence de toute tentative de sectorisation des zones cémétériales et de la permanence des traditions familiales en la matière, faisant apparaître la société de l'Empire romain tardif sous un jour différent, en fait bien plus sécularisée qu'on ne le pense généralement. Dans l'exposé qu'il consacre à l'implantation des manichéens en Égypte, Jean-Daniel Dubois présente sommairement les nouvelles ressources documentaires fournies par les recherches archéologiques menées sur le site de Kellis et les perspectives qui sont ouvertes par la mise au jour de ces éléments. Enfin, Christelle Jullien et Florence Jullien entreprennent, à travers l'étude des Actes de Mâr Mâri, de mettre en évidence les schémas identitaires propres aux communautés chrétiennes orientales, notamment dans le rapport à la fonction du missionnaire fondateur.

En conclusion, au-delà de l'impossibilité de construire une définition qui serait susceptible de s'appliquer à toutes les communautés considérées et qui se révélerait inévitablement trop générale et appauvrissante, John A. North insiste sur la nécessité d'intégrer l'évolution des communautés religieuses dans la compréhension générale des changements les plus importants dans la nature de la vie religieuse. Au terme de ces nombreuses contributions se dégagent trois principes de travail sur les communautés religieuses : la prise en compte de l'expérience individuelle parallèlement au développenent des communautés; l'impossibilité de séparer les différentes traditions religieuses du contexte dans lequel elles évoluaient et de l'influence qu'elles exerçaient les unes sur les autres; et la mise en œuvre d'une approche nuancée capable de rendre compte de l'évolution des communautés religieuses à travers les siècles dans le respect de tous les aspects contextuels.

D'un point de vue pratique, cet excellent ouvrage, qui n'a d'autre ambition que d'apporter des éléments de réponse et qui ouvre effectivement la voie à des questionnements ultérieurs sur des bases plus solides, contient, en plus clu résumé proposé pour chacune des contributions, un aperçu bibliographique parfois assez abondant. 